\title{
Effect of Pregabalin on Preoperative Anxiety and Postoperative Pain in Spine Surgery: A Randomized Controlled Study
}

\author{
Rahul Yadav ${ }^{1}$ Rajeeb K. Mishra ${ }^{1} \quad$ Arvind Chaturvedi ${ }^{1}$ Girija P. Rath ${ }^{1}$ \\ ${ }^{1}$ Department of Neuroanaesthesiology and Critical Care, All India \\ Institute of Medical Sciences, New Delhi, India \\ Address for correspondence Girija P. Rath, MD, DM, \\ Department of Neuroanaesthesiology and Critical Care, \\ Neurosciences Centre, All India Institute of Medical Sciences, \\ New Delhi 110 029, India (e-mail: girijarath@yahoo.co.in).
}

J Neuroanaesthesiol Crit Care 2018;5:8-14.

\begin{abstract}
Background Pregabalin is a gabapentinoid which has been shown to possess analgesic and anxiolytic properties. The present study aimed to assess the efficacy of a single dose of pregabalin on postoperative pain and preoperative anxiety in patients undergoing lumbar spine surgery.

Materials and Methods Sixty adults posted for elective lumbar laminectomy and discectomy were randomly assigned to one of the three groups; Group $C$ received placebo capsules, Group PI received pregabalin $150 \mathrm{mg}$, and Group P2 received pregabalin $300 \mathrm{mg}$ capsules, $2 \mathrm{~h}$ prior to induction of anesthesia. The levels of anxiety and pain were assessed with respective $100 \mathrm{~mm}$ visual analog scale (VAS) score. Postoperative fentanyl consumption and occurrence of side effects were recorded. All patients were observed for $8 \mathrm{~h}$, postoperatively.

Results There was a significant reduction in preoperative anxiety in both pregabalin groups as compared to control group $(p=0.001)$. VAS score for pain (at rest and during movement) was significantly decreased at all time intervals in both $\mathrm{Pl}$ and $\mathrm{P} 2$ groups as compared to control $(p<0.05)$. Fentanyl consumption in the postoperative period was significantly reduced in pregabalin groups as compared to control $(p=0.001)$. The level of sedation was higher in P2 group in the first $4 \mathrm{~h}(p<0.05)$. The occurrence of postoperative nausea and vomiting was more in control group as compared to the pregabalin groups $(p=0.018)$. The incidence of dizziness and blurring of vision was significantly more in $\mathrm{P} 2$ group.

Keywords

- fentanyl consumption

- postoperative pain

- pregabalin

- preoperative anxiety

Conclusion Single preoperative dose of pregabalin 150 or $300 \mathrm{mg}$ was effective in reducing preoperative anxiety, postoperative pain and total fentanyl consumption in patients undergoing lumbar laminectomy and discectomy. However, the incidence of postoperative side effects such as sedation, dizziness and visual blurring was more in patients who received pregabalin $300 \mathrm{mg}$.
\end{abstract}

\section{Introduction}

Despite better understanding of acute pain physiology over the past decade, approximately $80 \%$ of patients undergoing surgical procedures experience postoperative pain. ${ }^{1}$ Acute postoperative pain is a predictor of chronic pain syndromes as a result of surgery. ${ }^{2}$ Although opioids are important component of postoperative pain management, they are associated with side effects, ${ }^{3}$ and so, the multimodal analgesic approach has been recommended for the management of acute postoperative pain. ${ }^{4,5}$ Experimental models of neuropathic pain and inflammatory hyperalgesia have shown that $\gamma$-amino butyric acid analogues, such as gabapentin and pregabalin, have antinociceptive and antihyperalgesic
Dol https://doi.org/ 10.1055/s-0037-1616037. ISSN 2348-0548.
Copyright (@2018 Indian Society of Neuroanaesthesiology and Critical Care
License terms

()(1) $\Theta \circledast$ 
properties. It has been suggested that central neuronal sensitisation may result in amplification of postoperative pain, ${ }^{6}$ and that preoperative administration of gabapentin, before inflammatory trauma or surgical stimulation, may reduce the degree of central sensitisation. ${ }^{7}$ Compared with gabapentin, pregabalin has better pharmacokinetic properties and fewer drug interactions, due to an absence of hepatic metabolism. ${ }^{8}$ Apart from its analgesic potency, pregabalin possesses anxiolytic properties. ${ }^{9}$

The primary objectives of this study were to investigate the effect of pregabalin premedication on preoperative anxiety and to evaluate the effect of pregabalin premedication on postoperative pain scores (both stationary and movement related) and opioid (fentanyl) consumption in patients operated for lumbar laminectomy and discectomy. The prevailing hypothesis was, with the decrease in preoperative anxiety, the management of postoperative pain would be better if these patients were given oral pregabalin at a dose of 150 or $300 \mathrm{mg}$ as compared to placebo. The secondary objectives were to investigate the side effects of pregabalin administration in these patients and to study the efficacy of pregabalin premedication in attenuating/modifying the hemodynamic and bispectral index (BIS) responses during induction of anesthesia and orotracheal intubation.

\section{Materials and Methods}

This was a prospective, randomized, double-blind, placebo-controlled study. After obtaining approval from the institute's Ethics Committee, written informed consent was obtained from sixty adults of either sex between the age group of 18 and 65 years, belonging to the American Society of Anesthesiologists physical status I and II, with symptoms of nerve root compression, posted for elective lumbar laminectomy and discectomy, were included in the study. Patients who were unable to understand the operation of patient-controlled analgesia (PCA) device, with known allergy to pregabalin/gabapentin and/or fentanyl, any history of drug and/or alcohol abuse, intake of non-steroidal antiinflammatory drugs (NSAIDs) within $24 \mathrm{~h}$ prior to operation, impaired kidney function, taking sedatives or anticonvulsants and pregnant females were excluded from this study. The patients were randomized with computer-generated block randomisation, into three groups of 20 each, either to receive placebo (B-complex), pregabalin $150 \mathrm{mg}$ or pregabalin $300 \mathrm{mg}, 2 \mathrm{~h}$ before anesthetic induction. All patients received $0.2 \mathrm{mg}$ glycopyrrolate intramuscularly, $1 \mathrm{~h}$ before surgery. Patients' anxiety level was assessed by $100 \mathrm{~mm}$ visual analogue scale (VAS) in the ward before administration of the study drug. Assessment of the score was repeated in the preinduction room and in the operating room (OR). In the OR, patients' baseline pain intensity at rest and during movement (from supine to lateral position) was assessed using VAS for pain. Simultaneously, baseline values of heart rate (HR), mean arterial blood pressure (MABP), BIS, respiratory rate (RR) and oxygen saturation $\left(\mathrm{SpO}_{2}\right)$ were noted.
Anesthesia was induced with fentanyl $2 \mu \mathrm{g} / \mathrm{kg}$, propofol $1.5-2 \mathrm{mg} / \mathrm{kg}$ and tracheal intubation facilitated with rocuronium $1 \mathrm{mg} / \mathrm{kg}$. Anesthesia was maintained with nitrous oxide and oxygen in 2:1 ratio with isoflurane (end tidal $0.8 \%-1.2 \%$ ) and intermittent doses of rocuronium. Fentanyl was repeated as per requirement, at the discretion of the attending anesthesiologist. Mechanical ventilation was adjusted to keep the end-tidal $\mathrm{CO}_{2}$ concentration between 36 and $38 \mathrm{mmHg}$. Lactated Ringer's solution was used for maintenance requirements of fluids throughout the surgery. Monitoring during anesthesia consisted of continuous electrocardiogram, HR, $\mathrm{SpO}_{2}$, NIBP, BIS, airway pressure, temperature and end-tidal anesthetic concentrations. BIS sensors were attached as per instructions of the manufacturer and connected to Aspect-2000 BIS monitor (Aspect medical system, Natick, MA, USA). All parameters were recorded at $15 \mathrm{~min}$ interval. After intubation, the eyes and face were covered with cotton pads. Patients were positioned prone on a Wilson's frame, with head in neutral position over a horseshoe headrest. Under proper aseptic precaution, the surgical site was infiltrated with lignocaine $1 \%$ with adrenaline $(1: 200,000)$. At the end of the surgery, patients were turned supine. Neostigmine $50 \mu \mathrm{g} / \mathrm{kg}$ and glycopyrrolate $10 \mu \mathrm{g} / \mathrm{kg}$ were given to reverse the residual neuromuscular blockade. Trachea was extubated when the patient was fully awake.

On arrival to the intensive care unit, patient's pain intensity at rest and during movement was assessed using pain VAS. Simultaneously, values of $\mathrm{HR}, \mathrm{MABP}, \mathrm{RR}$ and $\mathrm{SpO}_{2}$ were noted and taken as the values at $0 \mathrm{~h}$. Then, the measurements were repeated at hourly intervals until $8 \mathrm{~h}$. Pain medications were converted to oral NSAIDs after the study period.

Patients were given a bolus dose of fentanyl $1 \mu \mathrm{g} / \mathrm{kg}$ through PCA pump. The incremental dose was set at $0.25-0.5 \mu \mathrm{g} / \mathrm{kg}$ with a lock-out interval of $10 \mathrm{~min}$ and $4 \mathrm{~h}$ limit of $0.4 \mathrm{mg}$. No background infusion was given. All patients received oxygen through face mask with a flow of $3 \mathrm{~L} / \mathrm{min}$ throughout the study period. A single observer, who was blinded to the groups, recorded all the measurements. Total dose of fentanyl consumed intra- and postoperatively over the $8 \mathrm{~h}$ period was noted. Sedation was assessed using Ramsay 5 point sedation score at every $2 \mathrm{~h}$ up to $8 \mathrm{~h}$. Adverse effects such as postoperative nausea and vomiting (PONV), dizziness, headache and visual blurring were noted.

\section{Statistical Analysis}

Sample size was computed to assess a mean difference of $15 \mathrm{~mm}$ in VAS score for pain between pregabalin groups and placebo group. With an assumption of standard deviation of $15 \mathrm{~mm}$ in each group, the required sample size for $\alpha$-error of 0.05 and power of $80 \%$ was estimated to be 16 individuals per group. We included twenty patients per group to cover for dropouts from the study. Outcomes were analyzed using oneway ANOVA for continuous variables and Kruskal-Wallis test for categorical variables. Postoperative fentanyl consumption was analyzed using one-way ANOVA and Fisher's exact test. The VAS for pain was compared by repeated-measures analysis followed by multiple comparisons with least square deviation method. The incidence of side effects was analyzed 
Table 1 Baseline characteristics

\begin{tabular}{|l|l|l|l|l|}
\hline Variable & Group C $(\boldsymbol{n}=\mathbf{2 0})$ & Group P1 $(\boldsymbol{n}=\mathbf{2 0})$ & Group P2 $(\boldsymbol{n}=\mathbf{2 0})$ & $\boldsymbol{p}$-Value \\
\hline Age $(\mathrm{y})$ & $41.6 \pm 11.5$ & $43.8 \pm 11.5$ & $40.8 \pm 11.0$ & 0.69 \\
\hline Weight $(\mathrm{kg})$ & $68.4 \pm 9.2$ & $66.5 \pm 10.3$ & $68.3 \pm 12.8$ & 0.83 \\
\hline Duration of anesthesia (min) & $171.3 \pm 50.0$ & $174 \pm 43.1$ & $173 \pm 41.2$ & 0.98 \\
\hline VAS score & $46.5 \pm 15.7$ & $17.5 \pm 6.4$ & $14.0 \pm 6.0$ & 0.001 \\
\hline MABP (mm Hg) & $107.4 \pm 9.5$ & $102.6 \pm 11.2$ & $100.3 \pm 8.2$ & 0.70 \\
\hline HR (beats/min) & $82.8 \pm 14.8$ & $81.6 \pm 11.6$ & $76.0 \pm 11.8$ & 0.21 \\
\hline BIS & $97.7 \pm 0.5$ & $97.7 \pm 0.5$ & $97.1 \pm 1.0$ & 0.008 \\
\hline
\end{tabular}

Abbreviations: BIS, bispectral index; HR, heart rate; MABP, mean arterial blood pressure; SD, standard deviation; VAS, visual analog scale. Note: All values expressed as mean $\pm \mathrm{SD}, p<0.05$ significant.

using Fisher's exact test. Statistical significance between the groups was analyzed using post hoc comparison (multiple comparisons) by Bonferroni's method. A $p$-value $<0.05$ was considered statistically significant. The statistical analysis was performed using the Statistical Package for Social Science software (SPSS for Windows, Version 15.0. Chicago, SPSS Inc.).

\section{Results}

A total of sixty patients enrolled and completed the study. The demographic characteristics such as mean age, weight, duration of anesthesia and hemodynamic parameters were comparable in all the three groups (- Table 1). The intergroup comparison revealed comparable ( $p=0.159$ ) VAS scores for anxiety in Groups P1 and P2; however, the VAS anxiety score was significandy lower in Group P1 and Group P2 as compared to Group C ( - Table 2 ).

\section{Intraoperative Hemodynamics}

Baseline and postinduction MABP and HR were comparable in the three groups (-Table 1). During the first 4 min after intubation, the increase in MBP and HR was significantly less in the P2 group ( - Table 3 ).

\section{Effect on Bispectral Index}

Baseline BIS was significantly less in Group P2, compared to Group C and Group P1 (- Table 1). Postinduction, it decreased in all the three groups, with the most significant decrease in Group P2 (- Table 4). Postintubation, an increase in BIS value was noticed in Group C ( - Table 5) only during the first $2 \mathrm{~min}$ $(p=0.001)$.

\section{Intraoperative Fentanyl Consumption}

Total intraoperative fentanyl consumption was significantly higher in Group C as compared to Group P1 and Group P2 whereas it was comparable in the P1 and P2 groups (-Table 6).

\section{Mean Visual Analog Scale Scores for Pain at Rest and Pain on Movement}

Both the mean VAS scores for pain at rest and pain on movement in the first $8 \mathrm{~h}$ were significantly less in the pregabalin groups (P1 and P2) as compared to placebo $(p=0.001)$.
However, they were comparable in P1 and P2 groups ( - Figs. 1 and $\mathbf{2}$ ).

\section{Total Postoperative Fentanyl Consumption}

Fentanyl consumption in the postoperative period was significantly reduced in P1 and P2 groups versus control group. However, it was comparable between P1 and P2 groups (-Table 6).

\section{Side Effects}

The level of sedation was higher in P2 group in the first $4 \mathrm{~h}$. The incidence of PONV was significantly more in control group compared to P1 and P2 groups ( $p=0.018$ ). Dizziness and blurring of vision were seen significantly in more number of patients in Group P2.

\section{Discussion}

The current concept of multimodal postoperative analgesia is mainly based on the combination of opioids, NSAIDs or paracetamol and perioperative administration of local anesthetics. The use of opioids may be limited by adverse effects, such as nausea, vomiting, excessive sedation, pruritus and urinary retention which can prolong the postoperative recovery period. NSAIDs are associated with damage to gastrointestinal mucosa, bleeding, renal toxicity, allergic reactions and heart failure. ${ }^{10}$ Cyclooxygenase- 2 selective NSAIDs may have prothrombotic properties, increasing the risk of stroke and myocardial ischemia. ${ }^{11}$ Anxiety is an unpleasant emotion and most patients awaiting elective surgery experience

Table 2 Intergroup comparison of visual analogue scale scores for anxiety

\begin{tabular}{|l|l|l|}
\hline Group $(\boldsymbol{n}=\mathbf{2 0})$ & VAS score & $\boldsymbol{p}$-Value \\
\hline Group C & $46.5 \pm 15.7$ & 0.001 \\
\hline Group P1 & $17.5 \pm 6.4$ & \\
\hline Group C & $46.5 \pm 15.7$ & 0.001 \\
\hline Group P2 & $14.0 \pm 6.0$ & \\
\hline Group P1 & $17.5 \pm 6.4$ & 0.16 \\
\hline Group P2 & $14.0 \pm 6.0$ & \\
\hline
\end{tabular}

Abbreviations: SD, standard deviation; VAS, visual analog scale.

Note: All values expressed as mean $\pm S D, p<0.05$ significant. 
Table 3 Mean arterial blood pressure and heart rate after induction

\begin{tabular}{|l|l|l|l|l|}
\hline Variable & Group C $(\boldsymbol{n}=\mathbf{2 0})$ & Group P1 $(\boldsymbol{n}=\mathbf{2 0})$ & Group P2 $(\boldsymbol{n}=\mathbf{2 0})$ & $\boldsymbol{p}$-Value \\
\hline MABP 0 & $116.5 \pm 15.1$ & $105.4 \pm 17.1$ & $96.3 \pm 16.4$ & 0.001 \\
\hline MABP 1 & $113.0 \pm 14.7$ & $105.8 \pm 17.6$ & $93.1 \pm 15.5$ & 0.001 \\
\hline MABP 2 & $103.8 \pm 10.2$ & $97.1 \pm 15.8$ & $89.6 \pm 14.0$ & 0.006 \\
\hline MABP 3 & $98.1 \pm 11.1$ & $90.9 \pm 12.3$ & $88.0 \pm 14.5$ & 0.04 \\
\hline MABP 4 & $94.9 \pm 9.9$ & $86.4 \pm 14.5$ & $85.1 \pm 14.1$ & 0.04 \\
\hline MABP 5 & $88.9 \pm 9.6$ & $83.6 \pm 13.6$ & $83.9 \pm 12.9$ & 0.30 \\
\hline MABP 10 & $85.0 \pm 6.6$ & $78.4 \pm 9.0$ & $78.2 \pm 9.9$ & 0.02 \\
\hline HR 0 & $109.1 \pm 18.8$ & $98.7 \pm 15.4$ & $89.8 \pm 18.4$ & 0.004 \\
\hline HR 1 & $110.2 \pm 16.1$ & $97.8 \pm 12.6$ & $89.7 \pm 16.7$ & 0.001 \\
\hline HR 2 & $102.7 \pm 13.5$ & $92.4 \pm 11.7$ & $87.3 \pm 17.9$ & 0.005 \\
\hline HR 3 & $100.3 \pm 14.6$ & $89.1 \pm 13.1$ & $85.9 \pm 19.0$ & 0.01 \\
\hline HR 4 & $95.5 \pm 13.5$ & $85.9 \pm 13.0$ & $83.6 \pm 19.7$ & 0.05 \\
\hline HR 5 & $91.1 \pm 13.8$ & $83.6 \pm 12.1$ & $81.5 \pm 18.9$ & 0.12 \\
\hline HR 10 & $82.0 \pm 12.7$ & $76.5 \pm 10.4$ & $75.0 \pm 13.9$ & 0.18 \\
\hline
\end{tabular}

Abbreviations: HR, heart rate; MABP, mean arterial blood pressure; SD, standard deviation.

Note: All values expressed as mean \pm SD, $p<0.05$ significant.

Table 4 Hemodynamic and bispectral index parameters after induction

\begin{tabular}{|l|l|l|l|l|}
\hline Variable & Group C $(\boldsymbol{n}=\mathbf{2 0})$ & Group P1 $(\boldsymbol{n}=\mathbf{2 9})$ & Group P2 $(\boldsymbol{n}=\mathbf{2 0})$ & $\boldsymbol{p}$-Value \\
\hline MABP $(\mathrm{mmHg})$ & $87.4 \pm 8.7$ & $86.3 \pm 8.8$ & $81.4 \pm 8.2$ & 0.068 \\
\hline HR (beats/min) & $87.0 \pm 13.7$ & $81.9 \pm 12.1$ & $76.2 \pm 14.03$ & 0.05 \\
\hline BIS & $59.9 \pm 5.9$ & $56.6 \pm 5.5$ & $55.4 \pm 4.9$ & 0.03 \\
\hline
\end{tabular}

Abbreviations: BIS, bispectral index; HR, heart rate; MABP, mean arterial blood pressure; SD, standard deviation.

Note: All values expressed as mean $\pm \mathrm{SD}, p<0.05$ significant.

Table 5 Bispectral index response after induction

\begin{tabular}{|l|l|l|l|l|}
\hline Variable & Group C $(\boldsymbol{n}=\mathbf{2 0})$ & Group P1 $(\boldsymbol{n}=\mathbf{2 9})$ & Group P2 $(\boldsymbol{n}=\mathbf{2 0})$ & $\boldsymbol{p}$-Value \\
\hline BIS 0 & $69.4 \pm 5.3$ & $63.2 \pm 5.9$ & $56.6 \pm 6.3$ & 0.001 \\
\hline BIS 1 & $71.2 \pm 5.2$ & $65.0 \pm 5.6$ & $57.5 \pm 6.2$ & 0.001 \\
\hline BIS 2 & $67.0 \pm 4.7$ & $63.1 \pm 4.2$ & $56.6 \pm 6.4$ & 0.001 \\
\hline BIS 3 & $63.2 \pm 5.1$ & $60.4 \pm 4.5$ & $55.6 \pm 6.3$ & 0.001 \\
\hline BIS 4 & $60.9 \pm 4.8$ & $58.5 \pm 4.3$ & $53.4 \pm 6.2$ & 0.001 \\
\hline BIS 5 & $59.2 \pm 4.7$ & $56.1 \pm 5.4$ & $53.2 \pm 5.8$ & 0.001 \\
\hline BIS 10 & $57.0 \pm 2.82$ & $53.2 \pm 5.2$ & $57.4 \pm 4.3$ & 0.001 \\
\hline
\end{tabular}

Abbreviations: BIS, bispectral index; HR, heart rate; SD, standard deviation.

Note: All values expressed as mean $\pm \mathrm{SD}, p<0.05$ significant.

preoperative anxiety. It may also adversely influence anesthetic induction and patient recovery, as well as decrease patient satisfaction with the perioperative experience.

Pregabalin has analgesic properties, ${ }^{12-15}$ opioid-sparing effects ${ }^{12,13}$ and relieves anxiety. ${ }^{14}$ Pregabalin probably reduces or modulates the release of excitatory neurotransmitters, ${ }^{2,16}$ leading to reduction in the level of anxiety and pain. Although pregabalin has been studied previously, a dose-ranging study has not been conducted in patients undergoing lumbar laminectomy and discectomy. In light of the previously published studies, we chose to evaluate the efficacy of pregabalin in doses of 150 and $300 \mathrm{mg}$ in patients undergoing lumbar laminectomy and discectomy, respectively. The minimum recommended dose of pregabalin has been advocated to be $150 \mathrm{mg}$, but not all the previous studies with $150 \mathrm{mg}$ pregabalin have shown a benefit. Therefore, we compared the dose response of 150 with $300 \mathrm{mg}$. In previous studies, pregabalin has been administered $1 \mathrm{~h}$ prior to induction of anesthesia. ${ }^{12-15}$ It has been observed that pregabalin displays a linear pharmacokinetics, and the time to peak plasma concentration is within 1 to $2 \mathrm{~h}$. The decision to administer pregabalin $2 \mathrm{~h}$ prior to induction of anesthesia was primarily to ensure that the peak plasma effect of pregabalin has been achieved at the time of assessing anxiety prior to induction of anesthesia. The inclusion of a placebo group in our study was to evaluate the influence of anesthetic technique on postoperative pain relief. In this study, 
Table 6 Intergroup comparison of total fentanyl consumption in intra- and postoperative period

\begin{tabular}{|l|l|l|}
\hline Group ( $\boldsymbol{n}=\mathbf{2 0})$ & Fentanyl $(\boldsymbol{\mu g})$ & $\boldsymbol{p}$-Value \\
\hline $\begin{array}{l}\text { Intraoperative } \\
\text { fentanyl consumption }\end{array}$ & \multicolumn{2}{l|}{} \\
\hline Group C & $201.5 \pm 46.4$ & 0.002 \\
\hline Group P1 & $151.8 \pm 39.2$ & \\
\hline Group C & $201.5 \pm 46.4$ & 0.04 \\
\hline Group P2 & $167.5 \pm 44.1$ & \\
\hline Group P1 & $151.8 \pm 39.2$ & 0.77 \\
\hline Group P2 & $167.5 \pm 44.1$ & \\
\hline $\begin{array}{l}\text { Postoperative } \\
\text { fentanyl consumption }\end{array}$ & $339.1 \pm 88.0$ & 0.001 \\
\hline Group C & $213.0 \pm 112.9$ & \\
\hline Group P1 & $339.1 \pm 88.0$ & 0.001 \\
\hline Group C & $190.8 \pm 45.3$ & \\
\hline Group P2 & $213.0 \pm 112.9$ & 1.00 \\
\hline Group P1 & $190.8 \pm 45.3$ & \\
\hline Group P2 & & \\
\hline
\end{tabular}

Abbreviation: SD, standard deviation.

Note: All values expressed as mean \pm SD, $p<0.05$ significant.

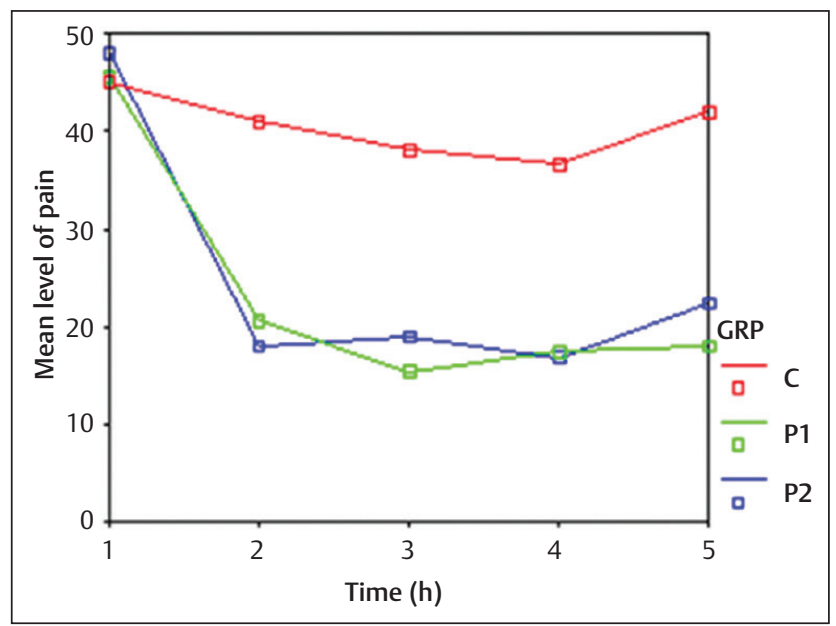

Fig. 1 Visual analog scale scores for pain at rest.

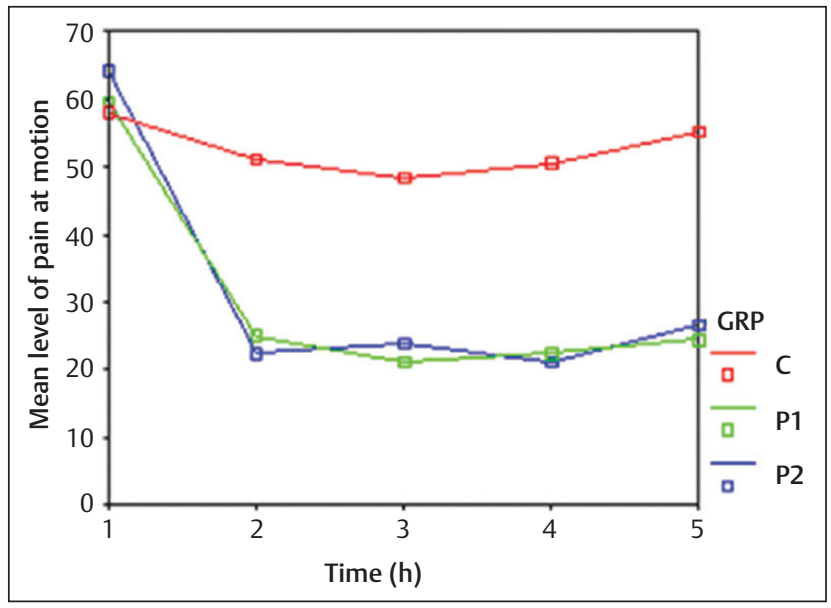

Fig. 2 Visual analog scale scores for pain on movement. the anesthetic regimen was standardised. Hence, the true protective effect of pregabalin could be revealed by comparing the analgesic outcome in the treatment and placebo groups.

In this study, it has been observed that preoperative administration of pregabalin in single dose of 150 and $300 \mathrm{mg}$ was effective in significantly reducing preoperative anxiety as compared to placebo. At the same time, all our patients were arousable and responding to commands (respiratory assessment score $\leq 3$ ). Earlier, it has been observed that the decrease in anxiety after premedication with pregabalin 150 and $300 \mathrm{mg}$ was similar to that of diazepam $5 \mathrm{mg}$ in day-care gynecological surgeries. ${ }^{14}$ On the contrary, White et $\mathrm{al}^{17}$ did not find any decrease in preoperative anxiety after administration of pregabalin at doses from $75 \mathrm{mg}$ to $300 \mathrm{mg}$. This could probably be due to the short time interval from administration of the study medication to induction of anesthesia in their study groups.

The mechanism by which pregabalin attenuates the pressor and BIS responses to laryngoscopy and intubation is unknown. Our results suggested that a single oral dose of pregabalin $300 \mathrm{mg}$ could significantly attenuate hemodynamic response to tracheal intubation compared to placebo and pregabalin $150 \mathrm{mg}$. Baseline BIS was significantly lower in the pregabalin $300 \mathrm{mg}$ group suggestive of increase in sedation/hypnosis level in this group even before induction. Induction of anesthesia expectedly decreased BIS in all the three groups, but the impact on BIS was greater in the pregabalin $300 \mathrm{mg}$ group. To our knowledge, no randomized controlled trial (RCT) has observed the effect of pregabalin premedication on hemodynamic and BIS response to laryngoscopy and tracheal intubation, till date.

It was also observed that pregabalin at doses of 150 and $300 \mathrm{mg}$ might cause a reduction in intraoperative opioid consumption. The total intraoperative fentanyl consumption in pregabalin group was significantly less as compared to placebo. On the contrary, all the previous studies have demonstrated no effect on total intraoperative analgesic use. ${ }^{15,17}$

In the present study, the mean VAS pain scores at rest and on movement were decreased by pregabalin premedication of 150 and $300 \mathrm{mg}$ as compared to placebo, suggesting that pregabalin effectively alleviates pain. The significant finding here is that pregabalin causes reduction in movement-evoked pain. These results are similar to the findings of Jokela et al in which they found that the area under curve for VAS scores for pain at rest, 1 to $8 \mathrm{~h}$ after surgery, and on movement were lower in the pregabalin $150 \mathrm{mg}$ group than that of placebo. ${ }^{14}$ Similarly, another RCT in patients undergoing laparoscopic cholecystectomy concluded that postoperative pain (at rest and on movement) scores were reduced in the pregabalin group in the first $24 \mathrm{~h}$ postoperatively. ${ }^{15}$ On the contrary, Mathiesen et al in abdominal hysterectomy patients, observed that pain scores remained similar for both pregabalin as well as placebo groups. ${ }^{18}$

In our study, premedication with both 150 and $300 \mathrm{mg}$ doses of pregabalin resulted in significantly less consumption of fentanyl in the first $8 \mathrm{~h}$, postoperatively. Many studies sought to determine whether perioperative pregabalin was effective in reducing postoperative pain 
and whether it had opioid-sparing effects. However, differences in the pregabalin dosages and types of surgery have yielded contrasting results. Reuben et al observed that, in patients undergoing decompressive lumbar laminectomy with posterior spinal fusion, pregabalin $150 \mathrm{mg}$ before and after surgery was as effective as celecoxib in reducing post-operative pain and patient-controlled morphine consumption, and the combination of both drugs was most effective. ${ }^{12}$ Mathiesen et al observed that pregabalin at the dose of $300 \mathrm{mg}$ causes 50\% reduction in $24 \mathrm{~h}$ postoperative morphine requirement. ${ }^{13}$ Jokela et al observed that analgesia was better during the first 8 h postoperatively, after premedication with pregabalin $150 \mathrm{mg}$ in patients undergoing day-care gynaecological laparoscopic surgeries. ${ }^{14}$ Agarwal et al in their study patients found that pregabalin premedication of $150 \mathrm{mg}$ is an effective method of reducing postoperative pain and fentanyl consumption. ${ }^{15}$ In patients undergoing laparoscopic cholecystectomy, administration of pregabalin $600 \mathrm{mg}$ significantly reduced postoperative pain and morphine consumption, at the cost of increased incidence of dizziness. ${ }^{16}$ On the contrary, White et al found that pregabalin at the doses of 75,150 , and $300 \mathrm{mg}$ did not cause any postoperative pain relief. ${ }^{17}$ This could probably be due to the nature of superficial surgical procedures with relatively low levels of pain in the postoperative period. Furthermore, patients were observed for only $2 \mathrm{~h}$ postoperatively whereas pregabalin has a biological half-life of 5.5 to $6.7 \mathrm{~h}$, and boluses of fentanyl were given to patients on complaining of moderate-to-severe pain instead of PCA. Peng et al observed that pregabalin $75 \mathrm{mg}$ provided limited analgesic benefit in the postoperative period. ${ }^{19}$

In our study, the incidence of PONV was significantly less in pregabalin groups as compared to placebo. Explanation for this could be due to decreased amount of fentanyl consumption in both intra- and postoperative period. In a study by Jokela et al, the authors observed that incidence of PONV was same in pregabalin $75,300 \mathrm{mg}$ groups and placebo group. ${ }^{14}$ Mathiesen et al found no difference in PONV in placebo and pregabalin $300 \mathrm{mg}$ groups. ${ }^{13,18}$ Agarwal et al also did not observe any difference in PONV between pregabalin $150 \mathrm{mg}$ and placebo groups in patients undergoing laparoscopic cholecystectomy. ${ }^{15}$

Dizziness and somnolence have been demonstrated to be the most common adverse effects of pregabalin in controlled studies of chronic pain. ${ }^{20}$ In our study, the incidence of pregabalin-related side effects such as dizziness and visual blurring was significantly more in pregabalin $300 \mathrm{mg}$ group compared to control and pregabalin $150 \mathrm{mg}$ groups. The level of sedation was also significantly higher in pregabalin $300 \mathrm{mg}$ group during the first $4 \mathrm{~h}$ postoperatively. Mathiesen et al observed increased sedation in patients receiving pregabalin $300 \mathrm{mg} .{ }^{13}$ Agarwal et al found that $150 \mathrm{mg}$ pregabalin was not associated with increased incidence of sedation and side effects. ${ }^{15}$

There are some limitations in our current study design. First, only a single dose of pregabalin was administered before surgery, and the maximum dose in our study was $300 \mathrm{mg}$. However, since the half-life of pregabalin is 5.5 to $6.7 \mathrm{~h}$, the effect of repeat doses of pregabalin remains to be studied. Second, we used a simple but well-validated measure of acute-state anxiety, namely the VAS score, because of the limited time available to perform the preoperative assessments. Clearly, more sophisticated psychological testing procedures might have been able to ascertain subtle and more specific effects of the drug on the patients' level of acute anxiety.

\section{Conclusion}

From this study, we conclude that pregabalin in both the doses given $2 \mathrm{~h}$ prior to the surgery can significantly reduce preoperative anxiety and intraoperative requirement of fentanyl compared to placebo. Both the doses of pregabalin significantly decreased postoperative pain VAS scores at rest and on movement during the first $8 \mathrm{~h}$ after surgery. Increased incidence of sedation, dizziness and visual blurring was observed more with pregabalin $300 \mathrm{mg}$ group, while reduced incidence of PONV was seen with either of the doses. Therefore, the single use of pregabalin in doses between 150 and $300 \mathrm{mg}$ is both safe and effective for reducing preoperative anxiety with a favourable postoperative analgesic profile.

\section{Funding \\ None.}

\section{Conflict of interest}

None.

\section{References}

1 Apfelbaum JL, Chen C, Mehta SS, Gan TJ. Postoperative pain experience: Results from a national survey suggest postoperative pain continues to be undermanaged. Anesth Analg 2003; 97:534-540

2 Perkins FM, Kehlet H. Chronic pain as an outcome of surgery. A review of predictive factors. Anesthesiology 2000;93:1123-33

3 Kehlet H, Wilmore DW. Multimodal strategies to improve surgical outcome. Am J Surg 2002;183:630-641

4 Kehlet H, Dahl JB. The value of "multimodal" or "balanced analgesia" in postoperative pain treatment. Anesth Analg 1993;77:1048-1056

5 White PF. Multimodal analgesia: Its role in preventing postoperative pain. Curr Opin Investig Drugs 2008;9:76-82

6 Woolf CJ, Chong MS. Preemptive analgesia - Treating postoperative pain by preventing the establishment of central sensitization. Anesth Analg 1993;77:362-379

7 Werner MU, Perkins FM, Holte K, Pedersen JL, Kehlet H. Effects of gabapentin in acute inflammatory pain in humans. Reg Anesth Pain Med 2001;26:322-328

8 Shneker BF, McAuley JW. Pregabalin: A new neuromodulator with broad therapeutic indications. Ann Pharmacother 2005;39:2029-2037

9 Lauria-Horner BA, Pohl RB. Pregabalin: A new anxiolytic. Investig Drugs 2003;12:663-672

10 Dolin SJ, Cashman JN. Tolerability of acute postoperative pain management: Nausea, vomiting, sedation, pruritus, and urinary retention. Evidence from published data. Br J Anaesth 2005;95: 584-591

11 Crofford LJ, Oates JC, McCune WJ, Gupta S, Kaplan MJ, Catella-Lawson F, et al. Thrombosis in patients with connective tissue 
diseases treated with specific cyclooxygenase 2 inhibitors. A report of four cases. Arthritis Rheum 2000;43:1891-1896

12 Reuben SS, Buvanendran A, Kroin JS, Raghunathan K. The analgesic efficacy of celecoxib, pregabalin, and their combination for spinal fusion surgery. Anesth Analg 2006;103:1271-1277

13 Mathiesen O, Jacobsen LS, Holm HE, Randall S, Adamiec-Malmstroem L, Graungaard BK, et al. Pregabalin and dexamethasone for postoperative pain control: A randomized controlled study in hip arthroplasty. Br J Anaesth 2008;101:535-541

14 Jokela R, Ahonen J, Tallgren M, Haanpää M, Korttila K. Premedication with pregabalin 75 or $150 \mathrm{mg}$ with ibuprofen to control pain after day-case gynaecological laparoscopic surgery. $\mathrm{Br} \mathrm{J}$ Anaesth 2008;100:834-840

15 Agarwal A, Gautam S, Gupta D, Agarwal S, Singh PK, Singh U. Evaluation of a single preoperative dose of pregabalin for attenuation of postoperative pain after laparoscopic cholecystectomy. Br J Anaesth 2008;101:700-704
16 Sarakatsianou C, Theodorou E, Georgopoulou S, Stamatiou G, Tzovaras G. Effect of pre-emptive pregabalin on pain intensity and postoperative morphine consumption after laparoscopic cholecystectomy. Surg Endosc 2013;27:2504-2511

17 White PF, Tufanogullari B, Taylor J, Klein K. The effect of pregabalin on preoperative anxiety and sedation levels: A dose-ranging study. Anesth Analg 2009;108:1140-1145

18 Mathiesen O, Rasmussen ML, Dierking G, Lech K, Hilsted KL, Fomsgaard JS, et al. Pregabalin and dexamethasone in combination with paracetamol for postoperative pain control after abdominal hysterectomy. A randomized clinical trial. Acta Anaesthesiol Scand 2009;53:227-235

19 Peng PW, Li C, Farcas E, Haley A, Wong W, Bender J, et al. Use of low-dose pregabalin in patients undergoing laparoscopic cholecystectomy. Br J Anaesth 2010;105:155-161

20 Kavoussi R. Pregabalin: From molecule to medicine. Eur Neuropsychopharmacol 2006;162):S128-S133 